\title{
Mobility-Aware Drop Precedence Scheme in DiffServ-Enabled Mobile Network Systems
}

\author{
Bongkyo Moon \\ Dongguk Univ-Seoul, Dept. of Computer Science and Engineering, \\ Seoul 100-715, South Korea \\ bkmoon@dongguk.edu
}

\begin{abstract}
In DiffServ-enabled mobile network systems, TCP sender may time out if packet loss occurs at handover event. Thus, TCP window size may be reduced temporarily due to packet loss. Under overloaded traffic situation in local IP-managed network, furthermore, the sending rate of TCP packets that a mobile host generates right after handover may not be enough to keep its contract service rate on SLA (service level agreement). Therefore, giving temporal priority to the packets in a handover flow can compensate for the reduction of packet sending rate after handover. In this paper, we propose a mobility-aware drop precedence scheme in order to alleviate the performance loss from temporal disconnection or the reduction of sending rate.
\end{abstract}

\section{Introduction}

In differentiated services (DiffServ) model, the RED (random early detection) mechanism is typically capable of dividing the available bandwidth fairly among TCP data flows that belong to the same AF (assured forwarding) PHB (perhop behavior) class, as packet loss automatically leads to a reduction of the packet sending rate in an TCP flow. In a RIO(RED with In and Out) queue, the packets of a flow are marked IN if the temporal sending rate at packet arrival is within the contract profile of the flow. Otherwise, the packets are marked OUT. The RIO mechanism actually starts to drop incoming OUT packets randomly with a certain probability in order to inform TCP sources of congestion after the average queue length of the buffer reaches the lower minimum threshold. It also starts to probabilistically drop IN packets when the average queue length exceeds the upper minimum threshold. However, due to the sawtooth variation of the TCP window, a flow has to transmit a certain amount of OUT packets in order to realize its reservation. In general, a connection with a larger reservation has a larger window, and hereby, it is obliged to transmit more OUT packets. Hence, it may not be easy to realize the reservation since OUT packets are very likely to be dropped. Until now, several studies have been done in order to solve this problem [1] 2] 3].

In a DiffServ-enabled mobile network system, meanwhile, the TCP sender may time out if packet loss occurs at handover event. Thus, TCP window size 
may be reduced temporarily due to the packet loss. Furthermore, if the managed IP network in the wireless mobile network architecture is overloaded and congested right after handover event, most of the low priority packets may be dropped in the same manner as the ordinary packets that belong to the same AF PHB class. Hereby, the packet-sending rate of TCP flow may be reduced again. Moreover, the fairness issue of the RED (random early detection) queue may not be temporarily kept, and the flow may fail to realize its reservation. In particular, in the case of streaming audio or video, service disruption might occur. Consequently, the sending rate of TCP packets that a MS (mobile station) generates right after handover may not be enough to keep its contract service rate on SLA (service level agreement) 10. Therefore, giving temporal priority to the packets of a handover flow can compensate for the reduction of packet sending rate in the managed IP network.

In this paper, we propose a mobility-aware drop precedence scheme in order to alleviate the performance loss from temporal disconnection or the reduction of sending rate. First, the DiffServ-based mobile network model is presented as an example. Second, a packet classification model for the mobility-aware drop precedence scheme is explained, and then AF PHB buffer with Markov chains is modelled. Finally the performance measures and results for the proposed scheme are presented.

\section{Mobile Network System Model}

\subsection{An Example of Mobile Network System}

In this section, we will consider the mobile WiMAX network as an example of the mobile network model. There are actually four main components in the mobile WiMAX network architecture: mobile station (MS), access service network - gateway (ASN-GW), connectivity service network (CSN), and core network. The Fig. 1 presents an example of the network architecture for Mobile WiMAX system. In this figure, the MS communicates with ASN-GW using wireless access technology, and also provides MAC processing functions, Mobile IP, authentication, packet retransmission, and handover. The ASN-GW also provides wireless interfaces for the MS, and takes care of wireless resource management, QoS support, and handover control. Hence, MSs can move efficiently between ASNGWs, provide smooth ASN-GWs transitions with minimal loss of packet data units (PDUs), and also provide fast ASN-GWs transition with guaranteed QoS. It means that when the MS roams from one ASN-GW to another, IP stack working on the top of L2 layer stays unaware on the roaming of MS. The CSN actually plays a key-role in IP-based data services including IP packet routing, security, QoS and handover control. In order to provide mobility for MS, the CSN supports handover between the ASN-GWs while Mobile IP provides handover between the CSNs [5] [6]. 


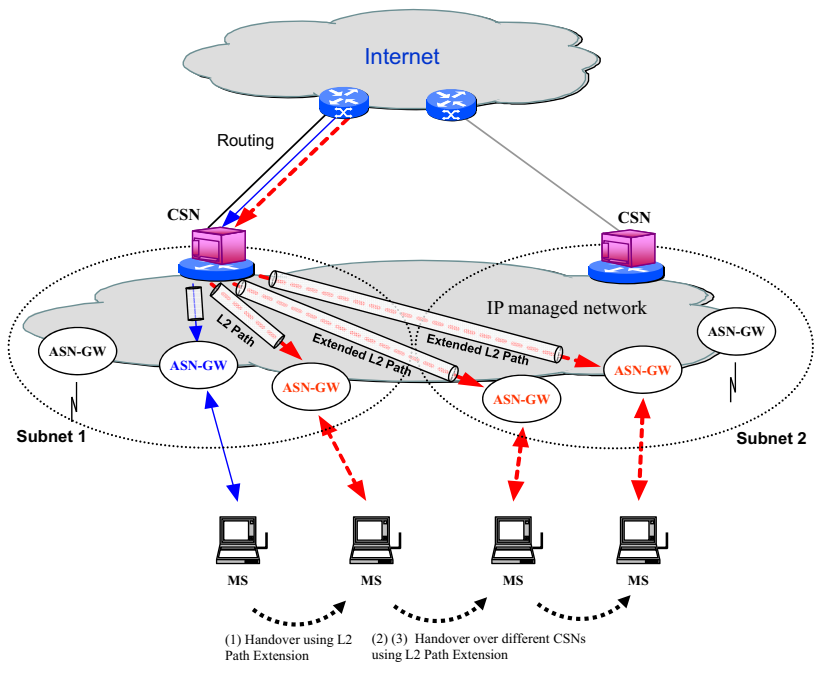

Fig. 1. Mobile WiMAX Network Diagram with Handover Scenario

\subsection{DiffServ Approach in Mobile Network System}

Fig. 2 shows a logical view of the DiffServ-based end-to-end IP QoS approach in Mobile WiMAX network architecture. In this figure, IPv6 packet is firstly encapsulated with 802.16 MAC header between MS and ASN-GW and then the encapsulation header is replaced with Ethernet header between ASN-GW and CSN. Meanwhile, an appropriate link path is set up through tunnel creation in order to meet the mobility requirements as shown in Fig. 1. Furthermore, the

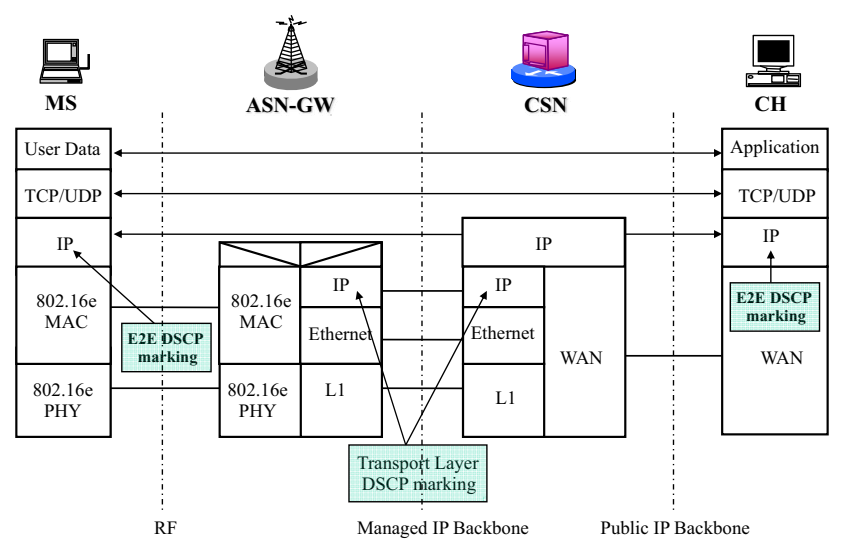

Fig. 2. DiffServ-based IP QoS approach in Mobile WiMAX Network Architecture 
mobility support by tunnel creation can make route path controlled over physically heterogeneous access network. Since the underlying routing path control due to mobility is actually independent from global IP layer, both IPv4 and IPv6 protocols can be used at the same time. For DiffServ in Mobile WiMAX network system, meanwhile, a new application flow which arrives in IP layer will be firstly parsed and classified according to the definition in DSCP (Diff-Serv Code Points). Eventually the flow is mapped into one of four types of services (UGS, rtPS, nrtPS or BE). For traffic classification and mapping strategies, AF rules are actually defined to map IP layer service into MAC layer services [7] [] [9].

\section{Mobility-Aware Drop Precedence Model}

Fig. 3 shows a simple DiffServ with AF PHB nodes and several traffic source groups in a mobile network system. In this figure, the CSN becomes a boundary node. It is essential to keep drop precedence policy of DiffServ model in L2 extension tunnel between CSN and ASN-GW in the managed IP network of Fig. 11. Here, two phase DiffServ mechanism for end-to-end QoS approach should be deployed. That is, the first phase should work between the first-hop router in local cloud, which the ASN-GW is directly connected to, and the boundary node (CSN). The second phase should work between the CSN and the $\mathrm{CH}$ (corresponding host) over the core network. In the second phase, actually the CSN becomes the global first-hop router.

We now assume that a MS is able to recognize the handover events and thus mark the DSCPs of packets it creates during handover. That is, the packets that a MS generates, during a handover period, can be marked by new mobilityaware (MA) tags with higher priority than the colours in three-drop precedence. In addition, when traffic exits from the managed IP network via a CSN, the packets marked with MA tag can be mapped to the normal three-drop precedence. Meanwhile, when a MS is a receiver, it informs the CSN of its location during handover in a mobile network system. After that, the CSN can re-mark the

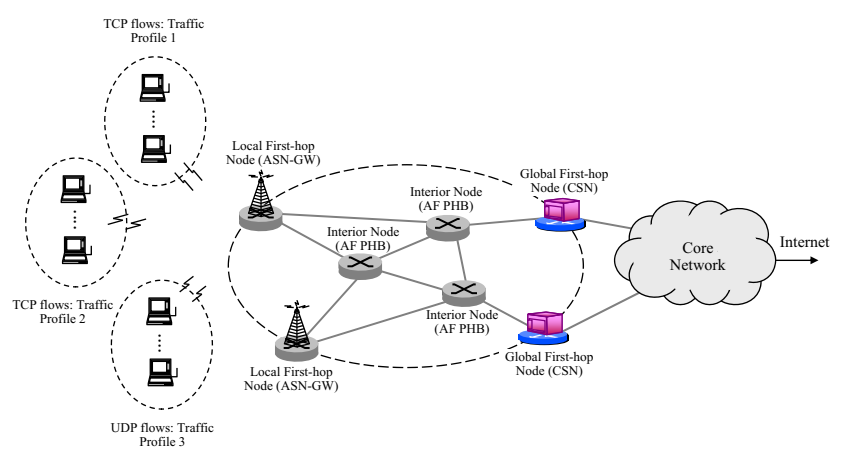

Fig. 3. Simple DiffServ Scenario with AF PHB nodes in Mobile Network System 


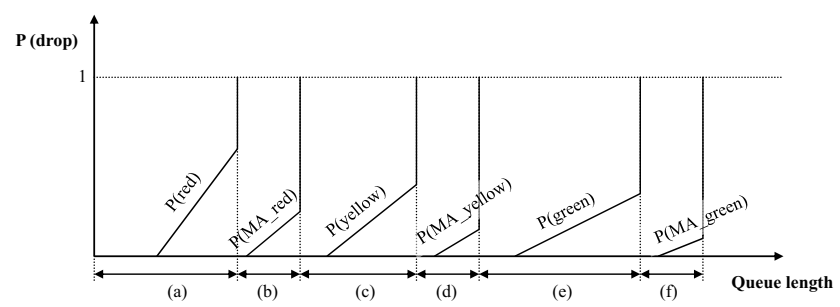

Fig. 4. Drop Probabilities of Mobility-aware Drop Precedence Scheme

incoming packets from the core network with MA tags in order to give priority to the handover flow.

In an MA drop precedence in managed IP network, there may be six possible subscription levels: 1) Only red packets are dropped [Fig. 4(a)]; 2) every red packet is dropped, and some of MA_red [Fig. 4(b)] packets are dropped; 3) every red and MA_red packet is dropped, and some yellow [Fig. 4(c)] packets are dropped; 4) every red, MA_red and yellow packet is dropped, and some of MA_yellow [Fig. 4(d)] packets are dropped; 5) every red ,MA_red, yellow and MA_yellow packet is dropped, and some green [Fig. 4(e)] packets are dropped; and 6) every red, MA_red, yellow, MA_yellow and green packet is dropped, and some MA_green [Fig. 4(f)] packets are dropped.

\section{Modelling of MA-Drop Precedence Scheme}

For simplicity of analytic model, one AF PHB node in a single link is considered. This model is used to compute steady state throughputs of the flows in the AF PHB node. The results can be used to analyze fairness and priority issues of the MA marking scheme. That is, the fairness issue includes how the AF link bandwidth is fairly shared between the different flows together with the MA marking in a mobile network system. The priority issue includes how the MA marking scheme can compensate mobility-sensitive TCP sources for the sending rate reduction that may be caused by handoff events. The analytic models in this section heavily rely on previous works [13]14.

\subsection{Packet Classifier Model}

The DSCP field provides the necessary information for classifying the packets at a DiffServ boundary node. In this section, the packet classifier is modelled with a flow conditioner mechanism which splits a flow into several subflows according to the levels of drop precedence. The subflows are marked with different levels of drop precedence depending on the traffic intensity characteristics and the handover rates of the flow.

Let $L=\left\{1, \ldots, N_{l}\right\}$ denote the set of TCP flow groups. Each flow group consists of $N_{l}$ identical flows. The sending rate and traffic profile associated with 
the flow together with the flow conditioner mechanism determine how a flow is split into different drop precedence levels. Let $x_{l}$ denote the sending rate of the flow associated with group $l$. Assume that flows in group $l$ are marked with the same AF class, and thus they are buffered in a queue in the AF PHB node. The flow conditioner splits the flow intensity into six drop precedence levels with the characteristic function $\alpha_{h}$, where $\alpha_{h}$ is 1 if handover occurs, otherwise it becomes 0 .

$$
\begin{aligned}
& \lambda_{l}^{(1)}= \begin{cases}\alpha_{h} x_{l}, 0 \leq x_{l} \leq v_{l}^{1} \\
\alpha_{h} v_{l}^{1}, v_{l}^{1} \leq x_{l} \leq v_{l}^{2} \\
\alpha_{h} v_{l}^{1}, x_{l} \leq v_{l}^{2}\end{cases} \\
& \lambda_{l}^{(2)}= \begin{cases}\left(1-\alpha_{h}\right) x_{l} & , 0 \leq x_{l} \leq v_{l}^{1} \\
\left(1-\alpha_{h}\right) v_{l}^{1} & , v_{l}^{1} \leq x_{l} \leq v_{l}^{2} \\
\left(1-\alpha_{h}\right) v_{l}^{1} & , x_{l} \leq v_{l}^{2}\end{cases} \\
& \lambda_{l}^{(3)}= \begin{cases}0 & , 0 \leq x_{l} \leq v_{l}^{1} \\
\alpha_{h}\left(x_{l}-v_{l}^{1}\right) & , v_{l}^{1} \leq x_{l} \leq v_{l}^{2} \\
\alpha_{h}\left(v_{l}^{2}-v_{l}^{1}\right) & , x_{l} \leq v_{l}^{2}\end{cases} \\
& \lambda_{l}^{(4)}= \begin{cases}0 & , 0 \leq x_{l} \leq v_{l}^{1} \\
\left(1-\alpha_{h}\right)\left(x_{l}-v_{l}^{1}\right) & , v_{l}^{1} \leq x_{l} \leq v_{l}^{2} \\
\left(1-\alpha_{h}\right)\left(v_{l}^{2}-v_{l}^{1}\right) & , x_{l} \leq v_{l}^{2}\end{cases} \\
& \lambda_{l}^{(5)}= \begin{cases}0 & 0 \leq x_{l} \leq v_{l}^{1} \\
0 & , v_{l}^{1} \leq x_{l} \leq v_{l}^{2} \\
\alpha_{h}\left(x_{l}-v_{l}^{2}\right) & , x_{l} \leq v_{l}^{2}\end{cases} \\
& \lambda_{l}^{(6)}= \begin{cases}0 & , 0 \leq x_{l} \leq v_{l}^{1} \\
0 & , v_{l}^{1} \leq x_{l} \leq v_{l}^{2} \\
\left(1-\alpha_{h}\right)\left(x_{l}-v_{l}^{2}\right) & , x_{l} \leq v_{l}^{2}\end{cases}
\end{aligned}
$$

The traffic profile of a flow is thus determined by two traffic intensity limits $v_{l}^{1}$ and $v_{l}^{2}, 0<v_{l}^{1}<v_{l}^{2}$. Let $\lambda_{l}^{(i)}$ denote the traffic intensity of the subflow that is marked with drop precedence $i$ within flow $l$. As a result, the characteristic function $\alpha_{h}$ and the traffic profile of the flow define $\lambda_{l}^{(i)}$ by equations (1) (6). The traffic conditioner definitions in equations (10) - (6) split a flow into drop precedence levels such that the perceptual distribution of a flow into drop precedence levels can be expressed by

$$
\delta_{l}^{(i)}=\frac{\lambda_{l}^{(i)}}{x_{l}}, \forall i \in I
$$

\subsection{AF PHB Buffer Model}

Fig. [5]shows an example of an AF PHB node with buffers in a 802.16e network. In this figure, $w 1, w 2, w 3$ and $w 4$ are weight factors for packet scheduling according to AF service classes. Each class buffer (FIFO queue) has its own threshold values according to drop precedence levels. In this model, however, only one class buffer is modelled in order to evaluate the effect of the number of threshold values in the proposed scheme. 
For simplicity of modelling, it is assumed that packets arrive according to a Poisson process and the packet service times are exponentially distributed. Even though this assumption is not suitable for exact modelling of packet arrival rate in the Internet, a Markov chain model can be used just for evaluating the performance of the proposed scheme compared to the existing three drop precedence scheme. Consequently, one class buffer can be modelled as a queueing system with a Markov model, and hereby, packet loss probabilities of subflows for each drop precedence aggregate can be computed.

Denote the acceptance thresholds for drop precedence level $i$ by $K_{i}, K_{1}=K$, in which $K$ is the size of the buffer. In the class 1 of the Fig. [5, therefore, $K_{1}$ is for MA_green, $K_{2}$ is for green, $K_{3}$ is for MA_yellow, $K_{4}$ is for yellow, $K_{5}$ is for MA_red, and $K_{6}$ is for red. Let $\lambda(i)$ denote the packet arrival rate into drop precedence class $i$ and $\mu^{-1}$ the mean value of the packet service time. Defining the cumulative sum of arrival intensities of drop precedence levels accepted into the buffer as $\lambda_{i}=\sum_{k=1}^{i} \lambda(k)$, the buffer can be modelled as an $M / M / 1 / K$ queue on the state space $\{m \mid 0 \leq m \leq K\}$, with state dependent arrival intensities. Thus, the stationary distribution of buffer occupancy can be solved from the balance equations of the system shown in (8). Let $\pi_{m}$ denote the equilibrium probability for state $m$. The balance equations for the one buffer behavior can be written as

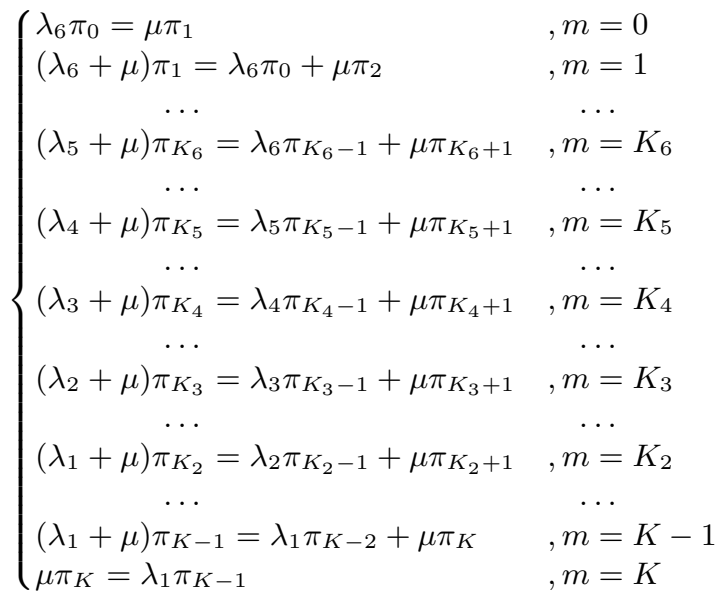

By solving the linear system of equations in (8), $\pi_{m}$ can be defined for $m=$ $1, \ldots, K$ as a function of $\pi_{0}$,

$$
\pi_{m}= \begin{cases}\left(\frac{\lambda_{6}}{\mu}\right)^{m} \pi_{0} & , 1 \leq m<K_{6} \\ \left(\frac{\lambda_{i-1}}{\mu}\right)^{m-K_{i}}\left(\prod_{j=i}^{5}\left(\frac{\lambda_{j}}{\mu}\right)^{K_{j}-K_{j+1}}\right)\left(\frac{\lambda_{6}}{\mu}\right)^{m} \pi_{0} & \left(i=2,3,4,5,6 \quad \text { and } \quad K_{1}=K\right)\end{cases}
$$

From the normalization condition $\sum_{m=0}^{K} \pi_{m}=1$, the equilibrium probability $\pi_{0}$ for the empty buffer state can be solved. As the buffer state probabilities $\pi_{m}$ are known, the packet drop probability $P(i)$ for packets aggregated to drop 


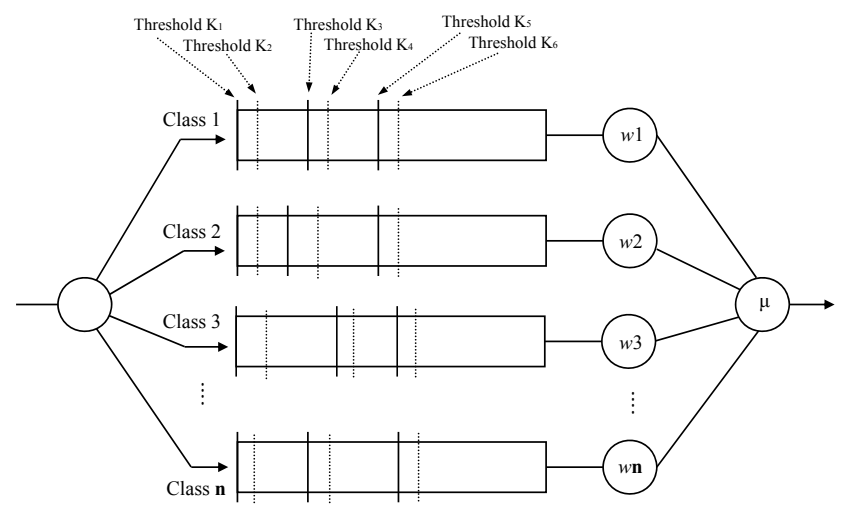

Fig. 5. Example of an AF PHB Node with n Buffers in a Mobile Network System

precedence $i$ can be solved by summing up the probabilities of states $m \geq K_{i}$,

$$
P(i)=\sum_{m=K_{i}}^{K} \pi_{m}
$$

\section{Performance Measures}

From the definition in equation (7), the total traffic intensity (packet arrival rate) of drop precedence level $i$ in a buffer with the same AF class can be obtained as

$$
\lambda(i)=\sum_{l} n_{l} \delta_{l}^{(i)} x_{l}
$$

Based on the packet drop probabilities $P(i)$ for drop precedence level $i$ from equation (10), the packet loss probability of a flow can be computed. The packet loss probability $q_{l}$ of a flow is defined as

$$
q_{l}=\sum_{i=1}^{6} \delta_{l}^{(i)} P(i) .
$$

It is assumed that TCP congestion control follows the differential equations for aggregates of TCP flows as described in [15. TCP throughput is thus proportional to average sending rate $x_{l}$ that depends on the round trip time (RTT) and the packet loss probability $q_{l}$ of a flow where

$$
x_{l}=\frac{1}{R T T} \sqrt{\frac{2\left(1-q_{l}\right)}{q_{l}}}, \quad l \in L_{T C P} .
$$

For each TCP group, equation (12) relates the TCP average sending rate with the packet drop probability via the AF PHB buffer. As the packet drop probability depends on the flow group $l$, the equation must be formulated for each TCP traffic profile group separately. 


\section{$6 \quad$ Results and Discussions}

In this section, one AF PHB class buffer model is used in evaluating how packet loss probability and average sending rate between the normal TCP flow and handover flow is achieved with the AF PHB mechanism. The buffer size is set to $K=39$ and the drop precedence limits for the buffer are $K_{3}=13, K_{2}=26$ and $K_{1}=39$. The model was evaluated with the buffer service intensities of both $\mu=1.0$ and $\mu=2.0$. The compensation for handover flow packets is implemented using reservation trunk. The buffer reservation rates $(R)$ for MA-tagged packets in the buffer model are fixed as both $30 \%$ and $60 \%$.

Fig. [6] shows the packet loss probability of handover flow in the AF PHB node as a function of packet sending rate with the buffer service intensity of $\mu=1.0$. In this figure, the MA-drop precedence scheme with reservation rate (e.g. $30 \%$ and $60 \%$ ) for handover flow has a smaller packet loss than the three-drop precedence scheme in the case where the load ratio (packet sending rate/buffer service intensity $\mu=1.0$ ) is less than 1.1. This is because the three-drop precedence does not provide any priority mechanism for handover flow. However, when the load ratio becomes considerably greater than 1.0, there is little difference in packet loss probability. This means that MA-drop precedence scheme does not get better results when network congestion level is high.

Fig. [7shows the packet loss probability of handover flow for a MA-drop precedence scheme when the buffer service intensity $\mu$ is 1.0 and 2.0. In this figure, when $\mu$ is 2.0, the MA-drop precedence scheme has much lower packet loss probability than when $\mu$ is 1.0 . In the cases when $\mu$ is 2.0 , and the load ratio approaches 1.0 (that is, when sending rate approaches 2.0), however, packet loss

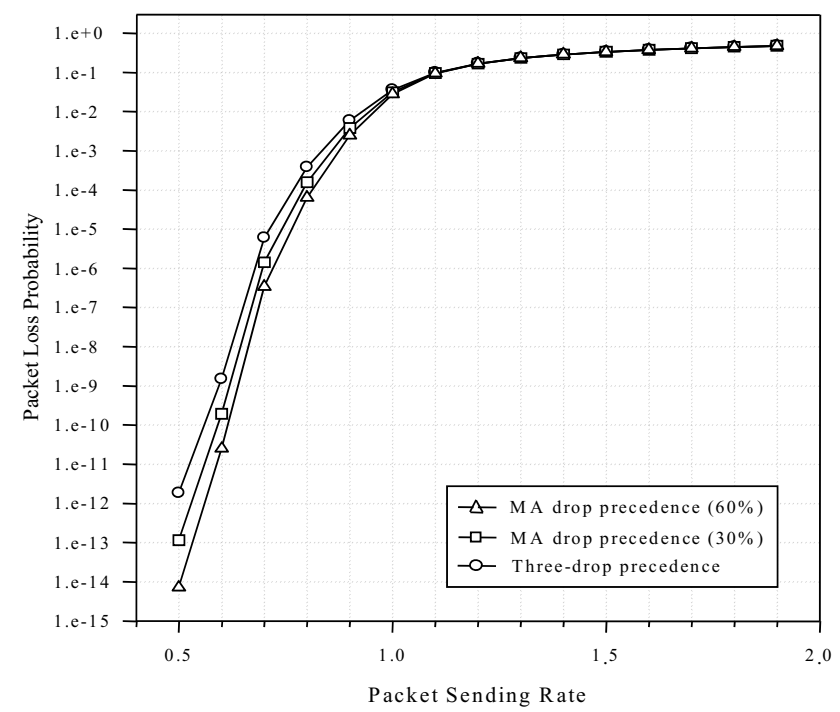

Fig. 6. Packet Loss Probability of Handover Flow in AF PHB Node $(\mu=1.0)$ 


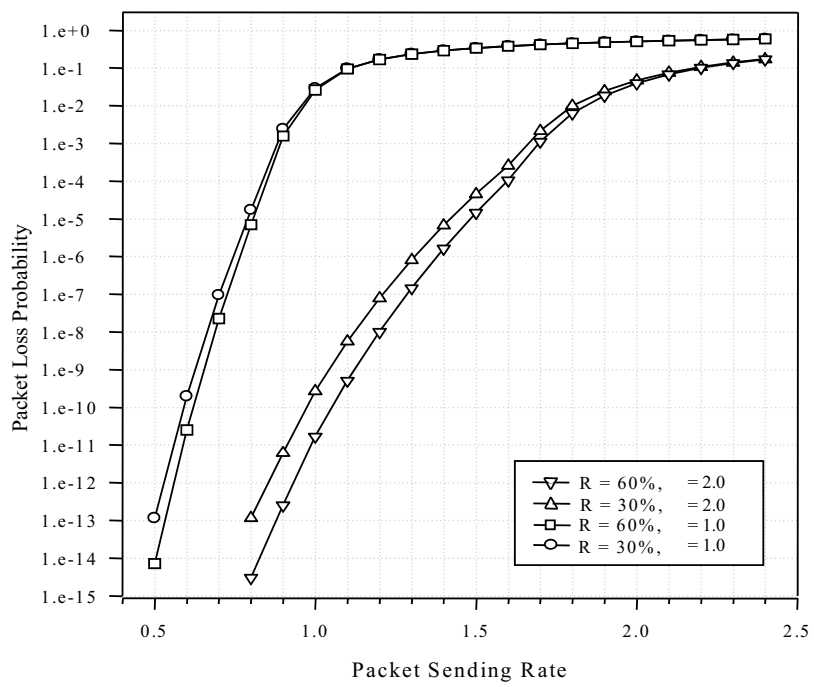

Fig. 7. Packet Loss Probability of Handover Flow in the AF PHB Node

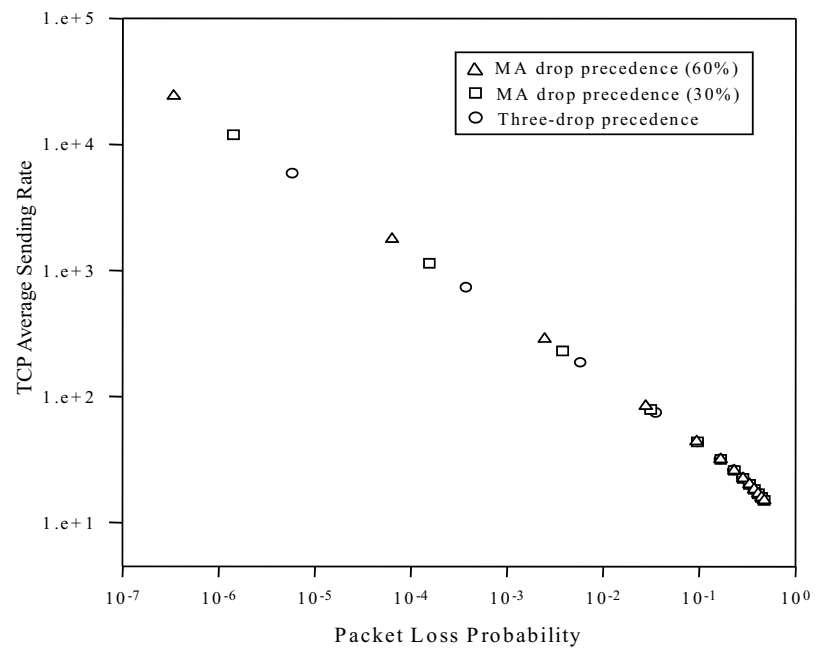

Fig. 8. Average Sending Rate of TCP Flow as a Function of Packet Loss Probability with $\mu=1.0$ and $R T T=0.1$

probabilities become nearly equal to each other although reservation rates for handover flow are both $30 \%$ and $60 \%$.

Fig. 8 shows the average sending rate (proportional to throughput rate) of a TCP flow as a function of packet loss probability with buffer service intensity $\mu=1.0$ and the round trip time $R T T=0.1$. Buffer reservation rates $(R)$ of both $30 \%$ and $60 \%$ are used for handover flow packets. In this figure, MA-drop 


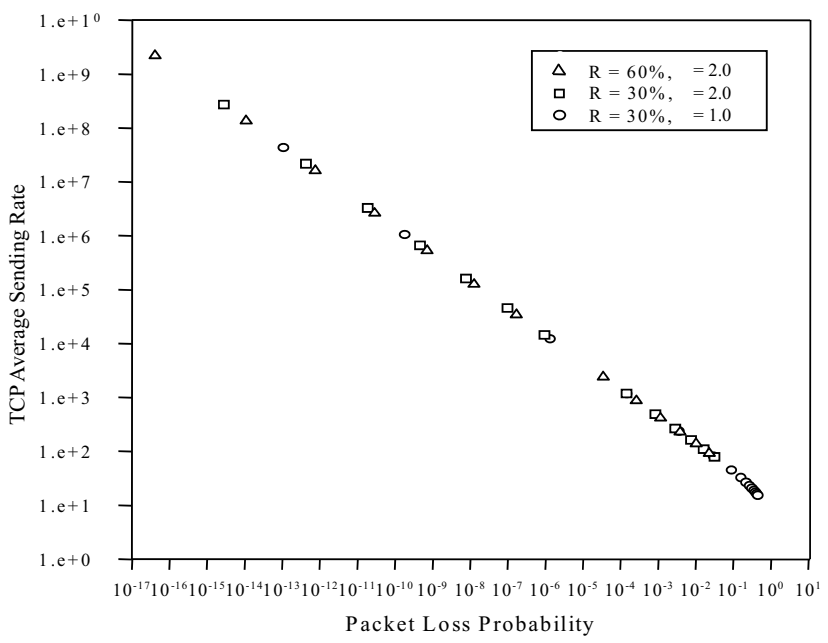

Fig. 9. Average Sending Rate of TCP Flow as a Function of Packet Loss Probability with $\mu=1.0$ and $\mu=2.0$, and $R T T=0.1$

precedence scheme for TCP handover flows can achieve much higher throughput rate than existing three-drop precedence scheme as long as packet loss probability is less than or equal to 0.1 . Moreover, the reservation rate of $60 \%$ gives higher throughput rates than that of $30 \%$. This result shows that the proposed scheme can compensate for the reduction of TCP window size due to frequent host mobility. However, a high reservation rate can make some buffers unused, and thus wasted, in the case where handover rate is low.

Fig. 9 shows that the reservation rate of $60 \%$ can, when $\mu$ is 2.0 , give much higher throughput rates than that of $30 \%$. On the other hand, a reservation rate of $30 \%$ with $\mu=1.0$ gives lower throughput rates compared to the case with $\mu=2.0$ and, in addition, most of them fall together in the area less than 50 in sending rate and greater than or equal to 0.1 in packet loss probability.

Consequently, when the network load is low, a TCP flow could achieve much higher throughput rate (proportional to average sending rate) than the contract rate. Hence, more packets in the flow are marked with a high drop precedence. Under more congested network environments, meanwhile, the sending rate of TCP flow tends to reduced less than the contract rate due to more packet loss. Hereby, the more packets in the flow can be marked into the low drop precedence levels. Therefore, when network congestion level is considerably low or high, packet differentiation in drop precedence is difficult to achieve.

\section{Conclusions}

In this paper, a mobility-aware drop precedence scheme is proposed in order to alleviate the reduction of sending rate in the managed IP network of mobile 
network systems. The proposed scheme could compensate for the reduction of TCP window size due to frequent mobility under wireless mobile environment. However, a high buffer reservation rate for the packets of handover flow can make some buffers unused and thus wasted in the case where handover rate is low. The proposed scheme is focused on the DiffServ-aware managed IP network of mobile network systems.

\section{Acknowledgements}

This work was supported by the Korea Research Foundation Grant funded by the Korean Government (MOEHRD, Basic Research Promotion Fund, KRF2006-331-D00357).

\section{References}

1. Feng, W., Kandlur, D., Saha, D., Shin, K.: Understanding TCP Dynamics in a Differentiated Services Internet. IEEE/ACM Transactions on Networking (April 1998)

2. Yeom, I., Narasimha Reddy, A.L.: Modeling TCP Behavior in a Differentiated Services Network. IEEE/ACM Transactions on Networking 1(9) (Feburary 2001)

3. Clark, D., Fang, W.: Explicit Allocation of Best Effort Packet Delivery Service. IEEE/ACM Transactions on Networking (August 1998)

4. Heinanen, J., Finland, T., Guerin, R.: A Two Rate Three Color Marker. Internet Draft (May 1999)

5. Andrews, J.G., Ghosh, A., Muhamed, R.: Fundamentals of WiMAX: Understanding Broadband Wireless Networking. Prentice Hall, Englewood Cliffs (2007)

6. WiMAX Forum, WiMAX End-to-End Network Systems Architecture (Stage 3), Network WG, January 11 (2008), http://www . wimaxforum.org/technology/documents

7. Chen, J., Jiao, W., Guo, Q.: An Integrated QoS Control Architecture for IEEE 802.16 Broadband Wireless Access Systems. Bell Labs Research China (2005)

8. Mai, Y.-T., Yang, C.-C., Lin, Y.-H.: Cross-Layer QoS Framework in the IEEE 802.16 Network. In: ICACT 2007, Feburary 2007, vol. 3, pp. 2090-2095 (2007)

9. Jiao, W., Chen, J., Liu, F.: Provisioning End-to-End QoS Under IMS Over a WiMAX Architecture. Bell Labs Technical Journal 12(1), 115-121 (2007)

10. Blake, S., Black, D., Carlson, M., Davies, E., Wang, Z., Weiss, W.: An Architecture for Differentiated Services. IETF RFC 2475 (December 1998)

11. Madanapalli, S., Patil, B., Nordmark, E., Choi, J., Park, S.: Transmission of IPv6 packets over 802.16's IPv6 Convergence Sublayer. IETF Internet-Draft (June 2006)

12. Jeon, H., Jeong, S., Riegel, M.: Transmission of IPv6 packets over Ethernet CS over IEEE 802.16 network. IETF Internet-Draft (September 2006)

13. Nyberg, E., Aalto, S., Virtamo, J.: Relating flow level requirements to DiffServ packet level mechanisms. COST279 TD (01) 04 (October 2001)

14. Kuumola, E.: Analytical Model of AF PHB Node in DiffServ Network. Mat-2.108, Networking Laboratory, Helsinki University of Technology (October 2001)

15. Kelly, F.: Mathematical Modelling of the Internet. In: Proceedings of Fourth International Congress on Industrial and Applied Mathematics, pp. 105-116 (1999) 